\title{
Neonatal Respiratory Dysfunction Caused by SFTPC Gene Mutation: a Case Report and Literature Review
}

\author{
Weijie $\mathrm{Yu}^{1}$, Qiuying $\mathrm{Hou}^{1}$, Qinlai Ying ${ }^{1}$, and Wen $\mathrm{Zhu}^{1}$ \\ ${ }^{1}$ The Second Hospital of Jiaxing
}

August 28, 2020

\begin{abstract}
Background: In this article, we present the case of a newborn with respiratory insufficiency caused by SFTPC gene mutation. We summarized and analyzed the clinical, chest imaging and gene test data of the case, and reviewed related literature. Case presentation: Case data: female infant, 6 hours with dyspnea. Physical examination on admission showed three concave signs, no rales or dryness in both lungs. Multiple chest radiographs indicated that the brightness of the two lungs gradually decreased, and chest CT indicated diffuse lung lesions. Sp-related gene detection indicated that SFTPC was newly mutated due to c.563t $>$ c (p.1188p). Literature reported six similar cases: 1) SFTPC gene c.68G > G/A, p.r23q heterozygosity missense mutation, 2) SFTPC gene c.115G > G/T, p.v39l heterozygosity missense mutation, 3) c.203T >a, p. Val68asp mutation, 4) c.435G > c mutation, 5) Cys121Phe/C121F mutation, and 6) p. Cp121gly /C121G mutation. All these cases developed severe neonatal respiratory distress syndrome shortly after birth, and there are no reports consistent with the gene loci and manifestations of this case. Conclusion: The mutation of SFTPC gene can cause early respiratory insufficiency and lead to progressive exacerbation of respiratory failure. We report a newly mutated SFTPC gene due to c.563t > c (p.1188p).
\end{abstract}

Neonatal Respiratory Dysfunction Caused by SFTPC Gene Mutation: a Case Report and Literature Review Weijie Yu, M.B.B.S., Qiuying Hou, M.B.B.S., Qinlai Ying, M.B.B.S., Wen Zhu*, M.B.B.S.

Pediatric department, the second affiliated hospital of Jiaxing medical college, Jiaxing children's medical center. Jiaxing city, Zhejiang province. 314200, China.

$*$ is the corresponding author.

Information of the corresponding author:

Mailing address: Pediatric department, the second affiliated hospital of Jiaxing medical college, Jiaxing children's medical center. Jiaxing city, Zhejiang province. 314200, China.

Telephone number: 0573-8208093

Fax number: 0573-82080930

E-maill address: edgar281@sina.com

Abstract

Background: In this article, we present the case of a newborn with respiratory insufficiency caused by SFTPC gene mutation. We summarized and analyzed the clinical, chest imaging and gene test data of the case, and reviewed related literature.

Case presentation: Case data: female infant, 6 hours with dyspnea. Physical examination on admission showed three concave signs, no rales or dryness in both lungs. Multiple chest radiographs indicated that 
the brightness of the two lungs gradually decreased, and chest CT indicated diffuse lung lesions. Sp-related gene detection indicated that SFTPC was newly mutated due to c.563t $>$ c (p.1188p). Literature reported six similar cases: 1) SFTPC gene c.68G > G/A, p.r23q heterozygosity missense mutation, 2) SFTPC gene c.115G > G/T, p.v39l heterozygosity missense mutation, 3) c.203T >a, p. Val68asp mutation, 4) c.435G > c mutation, 5) Cys121Phe/C121F mutation, and 6) p. Cp121gly /C121G mutation. All these cases developed severe neonatal respiratory distress syndrome shortly after birth, and there are no reports consistent with the gene loci and manifestations of this case.

Conclusion: The mutation of SFTPC gene can cause early respiratory insufficiency and lead to progressive exacerbation of respiratory failure. We report a newly mutated

SFTPC gene due to c.563t > c (p.1188p).

Keywords: SFTPC; genetic mutation; respiratory insufficiency

Introduction:

Genetic surfactant disorders often cause neonatal respiratory distress syndromes.

Pulmonary surfactant could prevent the lung alveoli from collapsing through reducing tension at the airwater interface. It consists of proteins and lipids. Half of proteins consist of four SPs, namely SP-A, SP-B, SP-C, and SP-D [1]. ABCA3, NKX2-1, GM-CSF are also involved in the process of surfactant generation and secretion. However, genetic variations are more frequently described in SFTPC and ABCA3, and less frequently in NKX2-1, SFTPB, SFTPA, and GM-CSF ${ }^{[2]}$. In this article, we present a case of the process of clinical diagnosis and treatment of a newborn with respiratory insufficiency caused by SFTPC gene mutation. The newborn developed aggravating symptoms. Chest imaging indicated diffuse lesions in both lungs. Sprelated gene detection indicated a novel mutation of SFTPC, which was newly mutated due to c.563t $>$ c (p.1188p).

Case History:

Case summary:

The newborn was female, G3P2, gestational age was 38 weeks +6 days. She was delivered by caesarean section due to "scarred uterus", birth weight was $3450 \mathrm{~g}$. The amniotic fluid was clear, umbilical cord and placenta were normal. Apgar score at 1 and 5 minutes were 8 and 9 points respectively. Half an hour after birth, she presented with shortness of breath and grunting. Thereafter, she was observed on oxygen for 6 hours. Without relief, she was transferred to our hospital.

Family history:

The parents were in good health and denied consanguineous marriages. There was no family history of hereditary diseases. The couple has a 7-year-old daughter without similar history.

Physical examination:

T: 37.5, P: 110 beats/min, R: 42 beats/min, conscious, slightly shortness of breath, moaning, the response cries were fine. The bregma was flat, $0.5 \times 0.5 \mathrm{~cm}$ in size. Neck was soft. In both lungs, respiratory sounds were coarse, a small amount of coarse wet rumbling was heard. Heart rhythms and heart sounds were normal, no murmurs heard. The abdomen was flat and soft, the liver was detected at $1.0 \mathrm{~cm}$ below the ribs, $1.5 \mathrm{~cm}$ below the xiphoid process, the splenic was not palpable, no mass was detected. The bowel sounds were present. Umbilical cord ligation was intact, and there was no exudate. Limb activity was less than expected, muscle tone was normal, limbs were cool.CRT was 3 seconds.

Auxiliary examination:

On admission, blood glucose was $3.9 \mathrm{mmol} / \mathrm{L}$, transcutaneous oxygen saturation was $100 \%$ (under nasal oxygen). 


\section{Lab tests:}

On Jan 1st, neonatal blood gas : PH was 7.34, partial pressure of carbon dioxide was $38.6 \mathrm{mmHg}$, partial pressure of oxygen was $145.4 \mathrm{mmHg}$, concentration of $\mathrm{HCO}_{3}$ was $20.4 \mathrm{mmol} / \mathrm{L}$, residual base was -4.87 $\mathrm{mmol} / \mathrm{L}$, oxygen saturation was $100.0 \%$, lactate was $1.90 \mathrm{mmol} / \mathrm{L}$. Pediatric routine blood test and hCRP test at emergency: white blood cell count was $32.64^{*} 10^{9} / \mathrm{L}$, ultra-sensitive C-reactive protein level was $3.58 \mathrm{mg} / \mathrm{L}$, percentage of neutrophil was $93.3 \%$, hemoglobin was $179 \mathrm{~g} / \mathrm{L}$, platelet count was $253^{*} 10^{9} / \mathrm{L}$. Emergency PCT: calcitonin level was $10.02 \mathrm{ng} / \mathrm{ml}$. On Jan 3rd ,neonatal blood gas : pH was 7.38 , partial pressure of carbon dioxide was $36.9 \mathrm{mmHg}$, partial pressure of oxygen was $32.9 \mathrm{mmHg}$, concentration of $\mathrm{HCO}_{3}$ was $21.4 \mathrm{mmol} / \mathrm{L}$, residual base was $-3.05 \mathrm{mmol} / \mathrm{L}$, oxygen saturation was $75.1 \%$, lactic acid was $3.2 \mathrm{mmol} / \mathrm{L}$, reduced hemoglobin was $24.3 \%$; On Jan 4th, neonatal blood gas : pH was 7.29, partial pressure of carbon dioxide was $53.4 \mathrm{mmHg}$, partial pressure of oxygen was $41.4 \mathrm{mmHg}$, concentration of $\mathrm{HCO}_{3}$ was $25 \mathrm{mmol} / \mathrm{L}$, residual base was $-2.48 \mathrm{mmol} / \mathrm{L}$, oxygen saturation was $80.4 \%$, glucose was $6.3 \mathrm{mmol} / \mathrm{L}$, lactic acid was $2.9 \mathrm{mmol} / \mathrm{L}$, reduced hemoglobin was $19.2 \%$; On Jan 6 th, neonatal blood gas : PH was 7.36 , partial pressure of carbon dioxide was $62.2 \mathrm{mmHg}$, partial pressure of oxygen was $46.0 \mathrm{mmHg}$, concentration of $\mathrm{HCO}_{3}$ was $34.2 \mathrm{mmol} / \mathrm{L}$, residual base was $6.65 \mathrm{mmol} / \mathrm{L}$, oxygen saturation was $85.7 \%$, reduced hemoglobin was $14.0 \%$, lactate was $1.1 \mathrm{mmol} / \mathrm{L}$. On Jan 7th, neonatal blood gas : PH was 7.37, partial pressure of carbon dioxide was $57.8 \mathrm{mmHg}$, partial pressure of oxygen was $75.1 \mathrm{mmHg}$, concentration of oxygen was $32.8 \mathrm{mmol} / \mathrm{L}$, standard bicarbonate concentration was $28.8 \mathrm{mmol} / \mathrm{L}$, total oxygen content was $75.1 \mathrm{mmol} / \mathrm{L}$, total carbon dioxide was $29.0 \mathrm{mmol} / \mathrm{L}$, residual base was $5.81 \mathrm{mmol} / \mathrm{L}$, oxygen saturation was $97.9 \%$, reduced hemoglobin was $14.0 \%$, lactic acid was $1.5 \mathrm{mmol} / \mathrm{L}$.

Imaging:

On Jan 1st, chest and abdomen x-ray illustrated that she had bronchitis. On Jan 4th, chest x-ray indicated that she had bronchiectasis.

Gene test:

Sp-related gene detection showed that SFTPC was newly mutated due to c.563t > c (p.1188p).

Treatment history:

After admission, the patient was placed in a warm box with nasal catheter oxygenation, then given cefotaxime injection and penicillin $\mathrm{G}$ sodium injection for anti-infection, given rehydration and other treatment to combat symptoms. On the third day after admission, under nasal catheter oxygenation $(0.5 \mathrm{~L} / \mathrm{min})$, there were repeated paroxysmal drop in oxygen saturation, which dropped to $60 \%-70 \%$ and could gradually rise again. On the fourth day, the paroxysmal drop was more frequent, and oxygen saturation dropped to $30 \%-70 \%$, then slowly rose on its own. There was no convulsion, no breath-holding, no apnea, no fever. She could drink milk. Physical examination showed that she had a concave sign, the cry was still loud, the response was normal. Morning TCB result: $3.9 / 3.4 \mathrm{mg} / \mathrm{dl}$. The respiratory sounds of both lungs were coarse, but no obvious rumbling was heard. Heart rhythm was normal, heart sounds were moderate, no murmur was heard. The abdomen was flat and soft, the liver was $1.0 \mathrm{~cm}$ below the rib, $1.5 \mathrm{~cm}$ below the xiphoid process, the splenic was not palpable, the bowel sounds were present, the limbs were less active, the extremities were warm. CRT was 3 seconds. On the eighth day, high-flow oxygen (Jan 8th-Jan 11th) and nebulization (Jan 8th-Jan 11th) were given, because of carbon dioxide retention in the follow-up blood gas analysis and low permeability of both lungs in chest radiograph, then changed to high-frequency assisted ventilation (Jan 11th to Jan 18th). On Jan 11th, $240 \mathrm{mg}$ of Curosurf was given as intra-tracheal drip, and then high-frequency assisted ventilation (Jan 18th -Jan 19th) was changed to normal-frequency assisted ventilation (Jan 19th-Jan 20th), because of positive inspiratory cavity, then changed to high-flow oxygen administration (Jan 20th-Feb 5th); On Jan 18th,chest CT suggested there were diffuse lung lesions, then underwent WES examination and bronchoscopic alveolar lavage. On Feb 2nd, follow-up chest radiograph showed that her lung transmittance decreased, again she was given Curosurf 480mg + Pulmicort 1mg via intra-tracheal drip. Dyspnea was improved for a while, then dyspnea worsened progressively. On Feb 5th, tracheal intubation was given, high-frequency ventilator-assisted ventilation was given until Feb 8th. The 
ventilator parameters gradually increased. Considering it was difficult to recover from the disease, the family gave up further treatments, then the patient died.

The chest radiograph gradually decreased in translucency as the disease progressed (Figure 1, Figure 2, Figure 3).

Discussion

Neonatal respiratory distress syndrome (NRDS) is one of the most common critical illnesses in the neonatal period, most often seen in premature infants, mainly due to small gestational age and inadequate production of surface active substances covering lungs. However, it could also be seen in full-term or near-full-term infants. The condition is often severe and is a common cause of neonatal death. NRDS is often treated with supplementation of lung surface active substances, sometimes the efficacy is not satisfying. The main components of pulmonary surfactant are SP and phospholipids, and SP contains four subtypes: SP-A, SP-B, SP-C and SP-D. Among them, SP-C is closely related to the development of respiratory distress syndrome (RDS) ${ }^{[3]}$. The activity and function of pulmonary surfactant are closely related to the concentration and activity of SP-C ${ }^{[4]}$. The SP-C gene is $3.5-\mathrm{kb}$ and it contains six exons, located on the short arm of chromosome 8 , which can selectively promote the synthesis of dipalmitoyl lecithin and phospholipid. It assists in the distribution of pulmonary surfactant on the alveolar surface and maintains its stability, reduces alveolar surface tension ${ }^{[4]}$. Alteration in the SP-C allele are closely related with NRDS. The mechanism is associated with the accumulation of misfolded SP-C precursor proteins. The misfolding could lead to protein aggregation and endoplasmic reticulum stress. Then cause pro-inflammatory cytokines releasing and lead to apoptosis ${ }^{[5-6]}$.

Genetic defects in surface active substance metabolism are related with a wide range of clinical manifestations ${ }^{[7]}$. Early treatment may improve symptoms, but diagnosis is often delayed due to phenotypic and genotypic variants. This case is a heterozygous variant of the SFTPC gene NM-003018: exon5: c.563T>C (p.L188P). The causative variant of SFTPC gene can cause pulmonary surfactant metabolism dysfunction type II; it is associated with progressive respiratory insufficiency and pulmonary disease, in which excessive lipoprotein accumulation in the alveoli causes severe respiratory distress. The main clinical features are neonatal respiratory insufficiency, alveolar protein deposition disease, and interstitial pneumonia. SFTPC mutations are caused by de novo mutations, they are autosomal dominant disorders and cases are mostly disseminated $^{[8]}$. Various types of SP-C gene mutations have been reported in the literature, with the exon 5 region being the most common mutation hotspot ${ }^{[9]}$. The pathogenesis of SFTPC mutation is the misfolding and abnormal processing of the SP-C precursor protein, which leads to cell damage and apoptosis, causing abnormal intracellular directional transport of SP-C protein and accumulation in alveolar type II cells ${ }^{[10-13]}$. Chronic alveolar inflammation is associated with interstitial lung disease in older children and adults [14]. Park et al reported the first case in Korea, a child who suffered neonatal RDS and developed childhood interstitial lung disease due to a novel heterozygous SFTPC mutation: c.203T $>\mathrm{A}^{[15]}$.Alzaid et al reported the first case in Arab world, with mutation of c.218T $>\mathrm{C}^{[16]}$.

Mutations in different sites of SFTPC cause variable clinical manifestations and transitions of associated lung disease, ranging from neonatal respiratory insufficiency, fatal neonatal respiratory distress syndrome, respiratory failure in infancy, interstitial lung disease in childhood, alveolar protein deposition, to chronic interstitial lung disease in adults. Children with mild disease gradually develop hypoxemia and dyspnea, and children with severe disease often die within 3 to 6 months, surviving children often need mechanical ventilation and long-term oxygen therapy, consistent with van Hoorn Jeroen ${ }^{[17]}$ and other reports. SFTPC mutations cause related pulmonary symptoms and treatment difficulties. Literature reported a case of SFTPC gene in exon 4 coding for Cys121Gly/C121G, experimental treatment with hydroxychloroquine has resulted in significant clinical improvement within 2 weeks $^{[18]}$. A case of successful lung transplantation has also been reported ${ }^{[19]}$. Early detection and diagnosis are relatively difficult in patients with early symptoms, and chest X-rays are not evident, high-resolution tomography (HRCT) scan features can help in early identification ${ }^{[20-21]}$. 
In summary, when neonates show signs of respiratory insufficiency such as repeated decreases in oxygen saturation after birth, especially in full-term infants, after excluding congenital heart disease, it is recommended to improve lung surface active substance-related gene testing. Early chest high-resolution CT examination can clarify the diagnosis and subsequent treatments could improve symptoms at an early stage.

Conclusion:

We reported a case with c.563t $>$ c (p.1188p) mutation of SFTPC gene. It can cause early respiratory insufficiency and eventually lead to progressive exacerbation of respiratory failure.

Declarations:

Ethics approval and consent to participate: The study was approved by Medical Ethics Committee of The Second Hospital of Jiaxing (Approval code: 20200522H03).

Availability of data and materials: Data sharing is not applicable to this article as no datasets were generated or analysed during the current study.

Competing interests: None declared.

Funding: Not applicable.

Acknowledgements: Not applicable.

Authors' contributions: Weijie Yu, Qiuying Hou, Qinlai Ying were responsible for the treatment of this patient, under the supervision of Yufeng Zhang and Wen Zhu. Weijie, Qiuying Hou did the literature review and drafted the manuscript.

List of abbreviation: CT: computated tomography, T: temperature, P: pulse, R: respiratory rate, CRT: clot retraction test, $\mathrm{HCO}_{3}$ : bicarbonate, TCB: total conjugated bilirubin.

References:

Akella A,Deshpande SB. Pulmonary surfactants and their role in pathophysiology of lung disorders[J].Indian J Exp Biol,2013,51(1):5—22.

Glasser R,Mallampalli K. Surfactant and its role in the pathobiology of pulmonary infection.[J] .Microbes Infect., 2012, 14: 17-25.

Sweet G, Carnielli V, Greisen G et al. European Consensus Guidelines on the Management of Respiratory Distress Syndrome - 2019 Update.[J] .Neonatology, 2019, 115: 432-450.

Jiang M,Roth G,Chun-On P et al. SFTPC Phenotypic Diversity Caused by Differential Expression of -CreTransgenic Alleles.[J] .Am. J. Respir. Cell Mol. Biol., 2020, 62: 692-698.

Almlén A,Walther J,Waring J et al. Synthetic surfactant based on analogues of SP-B and SP-C is superior to single-peptide surfactants in ventilated premature rabbits.[J] .Neonatology, 2010, 98: 91-9.

Nathan N, Borensztajn K, Clement A. Genetic causes and clinical management of pediatric interstitial lung diseases.[J] .Curr Opin Pulm Med, 2018, 24: 253-259.

Delestrain C, Simon S ,Aissat A et al. Deciphering the mechanism of Q145H SFTPC mutation unmasks a splicing defect and explains the severity of the phenotype.[J] .Eur. J. Hum. Genet., 2017, 25: 779-782.

Da H,Yuanyuan Q,Huijun W et al. Surfactant protein C gene mutations in two newborns with neonatal respiratory distress syndrome and literature review $[\mathrm{J}]$. Chinese Journal of Evidence -Based Pediatric, 2016, 11(1): 51-55.

Chen J H,Zhao D Y,An S H et al. [Clinical manifestations of three cases of surfactant protein C p. V39L mutation].[J] .Zhonghua Er Ke Za Zhi, 2017, 55: 457-461. 
Liu T,Sano K , Ogiwara N et al. A novel surfactant protein C L55F mutation associated with interstitial lung disease alters subcellular localization of proSP-C in A549 cells.[J] .Pediatr. Res., 2016, 79: 27-33.

Hawkins A,Guttentag H,Deterding R et al. A non-BRICHOS SFTPC mutant (SP-CI73T) linked to interstitial lung disease promotes a late block in macroautophagy disrupting cellular proteostasis and mitophagy.[J] .Am. J. Physiol. Lung Cell Mol. Physiol., 2015, 308: L33-47.

Katzen J,Wagner D,Venosa A et al. An SFTPC BRICHOS mutant links epithelial ER stress and spontaneous lung fibrosis.[J] .JCI Insight, 2019, 4: undefined.

Hong D, Qi Y, Liu J, et al. A novel surfactant protein C mutation resulting in aberrant protein processing and altered subcellular localization causes infantile interstitial lung disease[J]. Pediatric Research.

Avital A,Hevroni A,Godfrey S et al. Natural history of five children with surfactant protein C mutations and interstitial lung disease.[J] .Pediatr. Pulmonol., 2014, 49: 1097-105.

Park S,Choi J,Kim T et al. SFTPC Pediatric Case Report on an Interstitial Lung Disease with a Novel Mutation of Successfully Treated with Lung Transplantation.[J] .J. Korean Med. Sci., 2018, 33: e159.

Alzaid A,Eltahir S,Amin M et al. SFTPC An gene mutation causes childhood interstitial lung disease: first report in the Arab region.[J] .JRSM Open, 2020, 11: 2054270419894821.

van Hoorn J,Brouwers A,Griese $\mathrm{M}$ et al. Successful weaning from mechanical ventilation in a patient with surfactant protein $\mathrm{C}$ deficiency presenting with severe neonatal respiratory distress. [J] .BMJ Case Rep, 2014, 2014: undefined.

Hepping N,Griese M,Lohse P et al. Successful treatment of neonatal respiratory failure caused by a novel surfactant protein C p.Cys121Gly mutation with hydroxychloroquine. [J] .J Perinatol, 2013, 33: 492-4.

Park S,Choi J,Kim T et al. SFTPCPediatric Case Report on an Interstitial Lung Disease with a Novel Mutation of Successfully Treated with Lung Transplantation.[J] .J. Korean Med. Sci., 2018, 33: e159.

Mechri M,Epaud R,Emond S et al. Surfactant protein C gene (SFTPC) mutation-associated lung disease: high-resolution computed tomography (HRCT) findings and its relation to histological analysis.[J] .Pediatr Pulmonol, 2010, 45: 1021-9.

Owen S,Manley J,Davis G et al. The evolution of modern respiratory care for preterm infants. $[\mathrm{J}]$.Lancet, 2017, 389: 1649-1659. 


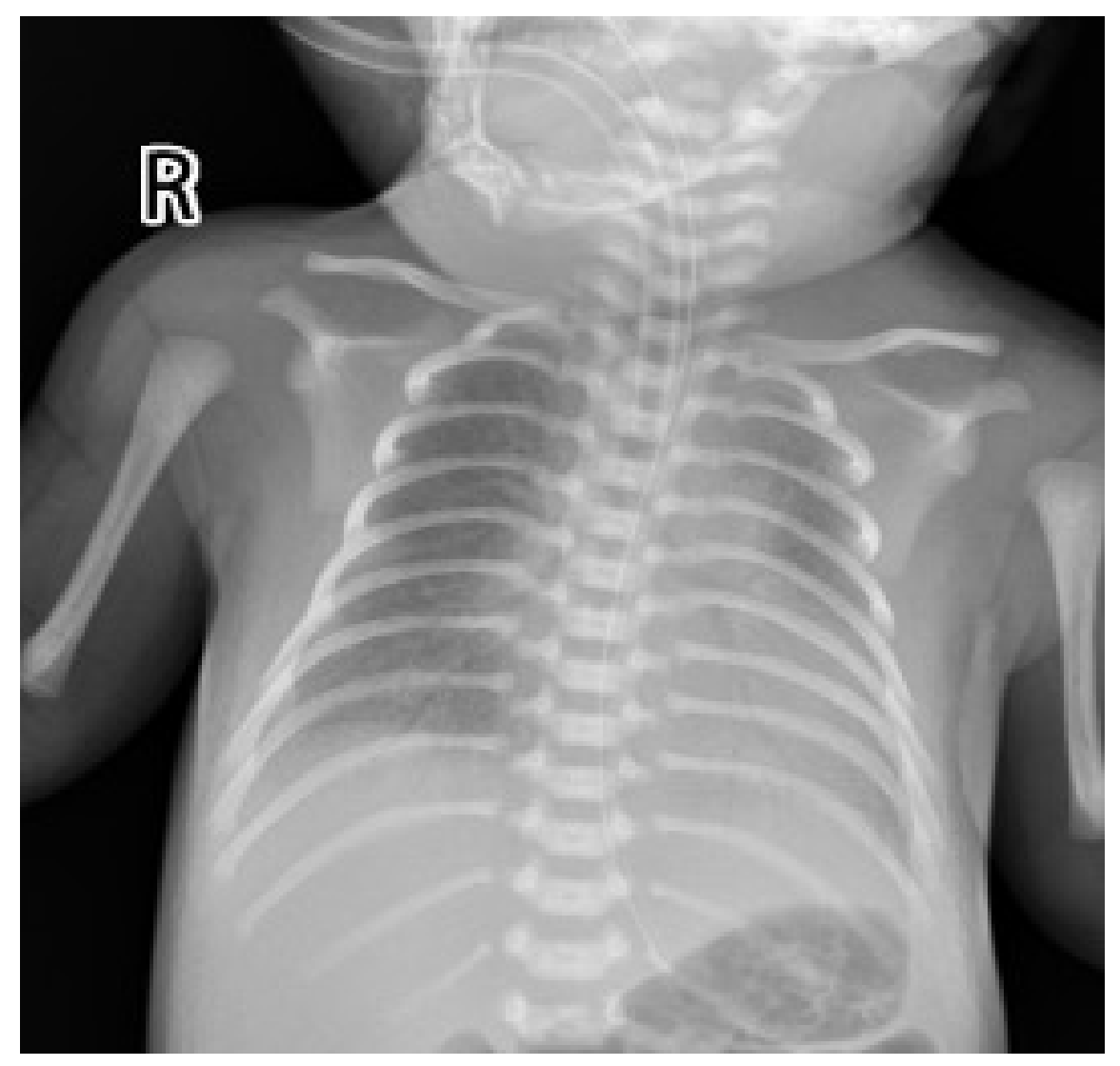




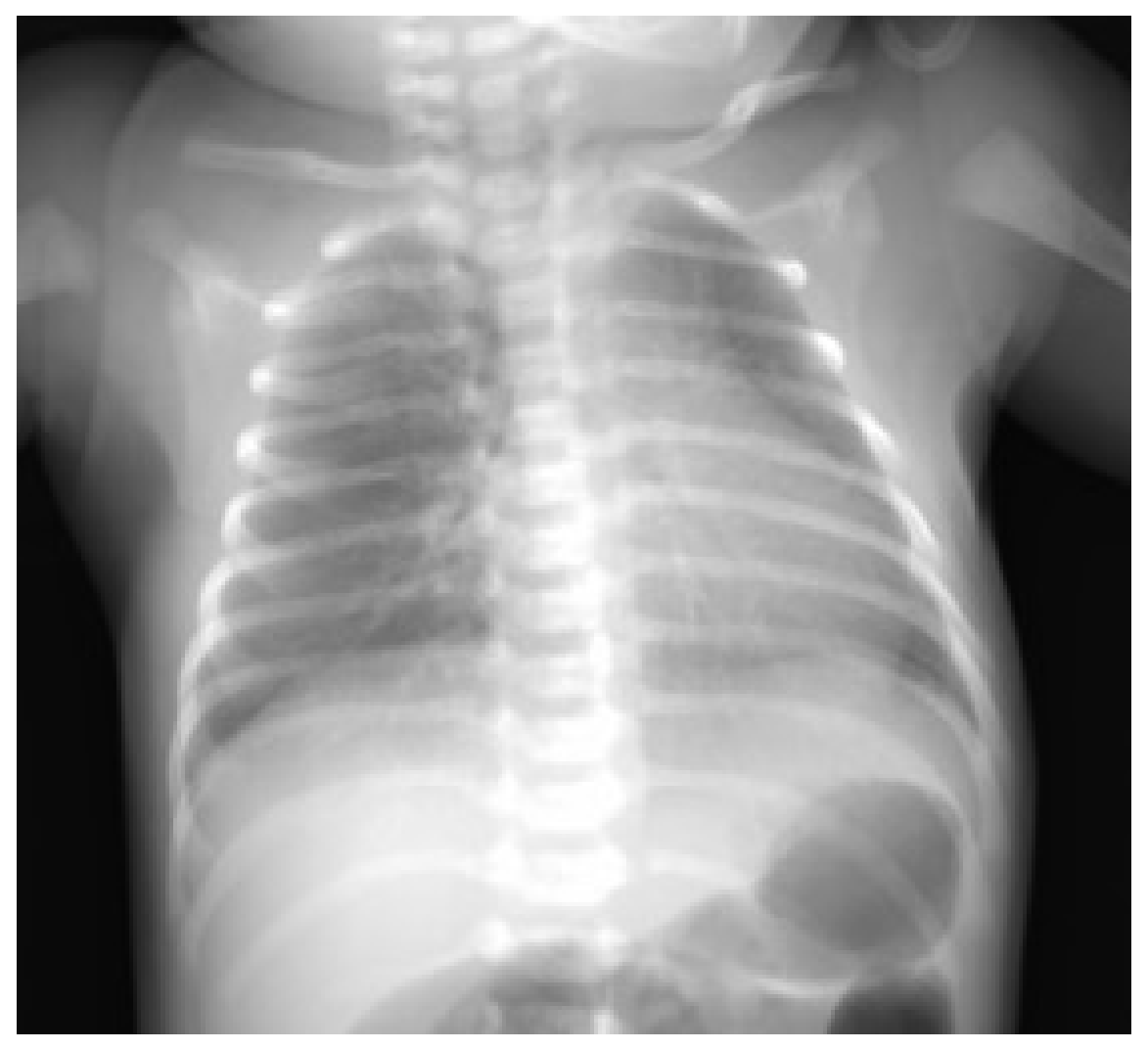




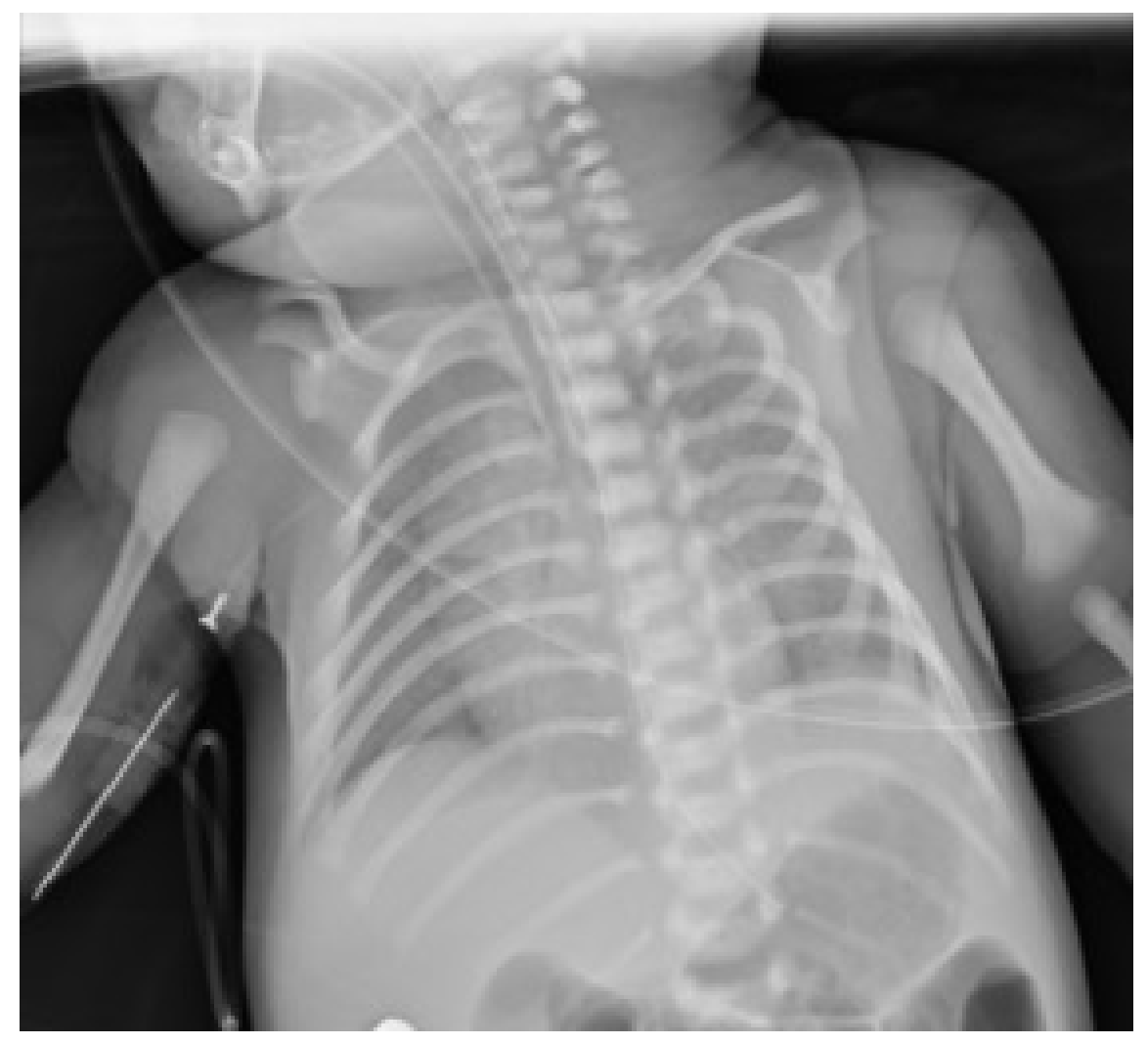

